\title{
Autonomia como categoria central no conceito de promoção de saúde
}

\section{Autonomy as a central criterion in the concept of health promotion}

Paulo Fleury-Teixeira ${ }^{1}$

Fernando Antônio Camargo Vaz ${ }^{1}$

Francisco Carlos Cardoso de Campos ${ }^{1}$

Juliana Álvares ${ }^{1}$

Raphael Augusto Teixeira Aguiar ${ }^{1}$

Vinícius de Araújo Oliveira ${ }^{1}$

\footnotetext{
${ }^{1}$ Núcleo deEstudosem SaúdeColetiva, Faculdade deM edicina, Universidade Federal de M inas Gerais. Av. Alfredo Balena 190, Santa Efigênia. 30130-100. Belo H orizonte M G paulofleury@medicina.ufmg.br
}

Abstract This article presents the central concepts of the multiple case study "H ealth Promotion in Primary Care" conducted by N esconUFM G in 2005. Teams of the Family Health Program developed twelve health promotion experiences in different Brazilian regions. After a conceptual revision and field research, health promotion was defined as the process of improving the health of people independently of any previous disease or injury, helping them to increase control over their health. We also concluded that an increased autonomy, understood as the capacity and possibility of the persons to choose, is the central category and criterion for defining health promotion practices.

Key words Health promotion, Autonomy, Primary care
Resumo Este artigo expõe os conceitos centrais do estudo demúltiplos casos: "Promoção deSaúde na Atenção Básica". 0 estudo foi conduzido pelo N escon-UFM G em 2005 em doze experiências de promoção da saúde realizadas por equipes do Programa de Saúde da Família (PSF) em diversas regiões do Brasil. A pós revisão teórica e pesquisa de campo, a definição de promoção da saúde adotada foi a atuação para a melhoria da saúde, anterior e independente a qualquer patologia ou agravo, ampliando-se o controle das pessoas sobre a própria saúde. Também concluímos que a ampliação da autonomia, vista como ampliação das capacidades e possibilidades de escolha das pessoas, éa categoria central eo critério definidor da atuação em promoção de saúde.

Palavras-chave Promoção de saúde, Autonomia, Atenção básica 
Introdução

Este artigo expõe o marco conceitual da pesquisa: "Promoção de Saúde na Atenção Básica no Brasil ${ }^{1 "}$, realizado pelo Nescon-UFM G, a pedido e sob patrocínio do Departamento de Atenção Básica (DAB/MS) e da Organização Panamericana de Saúde - OPS/OM S - representação Brasil, em 2005. A pesquisa consistiu em estudo descritivo de múltiplos casos, sobre experiências de promoção de saúde desenvolvidas por equipes do Programa deSaúde da Família (PSF), em quatro regiões do Brasil.

A seleção dos casos se deu através da análise de mais de 1.800 relatos de experiências em PSF apresentados no VII Congresso Brasileiro de Saúde Coletiva, organizado pela Associação Brasileira de Pós-graduação em Saúde Coletiva ABRASCO, realizado entre 29 de Julho e 02 de Agosto de $2003^{2}$, edemais de 1.600 exposiçõesna II M ostra $N$ acional de Saúde da Família ${ }^{3}$ realizada entre 1 e 3 dejunho de 2004.

Foram selecionadas e estudadas as seguintes experiências:

. Projeto de desenvolvimento infantil de Santana do Aurá - comunidade de catadores de lixo próxima ao aterro sanitário de Belém, Pará;

- Programa de Saúde da Família no Distrito Sanitário de M osqueiro, Belém, Pará;

. Conquistando o Futuro, em Pedreira, São Paulo;

Relato de uma experiência de gênero com travestis na atenção básica à saúde e resgate da cidadania - GATASS, Recife, Pernambuco;

- Proposta Municipal de Educação Popular em Saúde - PM EPS, Recife - Pernambuco;

. Projeto Alfabetizante com Amor. Itambé, Pernambuco;

- Conselho local de saúde: Ampliando ações na comunidade, Blumenau, Santa Catarina;

- Cuidando da Saúde da Família Guarani, Viamão, Rio Grande do Sul;

. Promoção à saúde e educação: planejamento participativo em território de programa de saúde da família, M ontes Claros, M inas Gerais;

Spa comunitário: uma experiência de reeducação alimentar e física no PSF, Santa Cruz, Rio Grande do Norte;

Gestão Ambiental Integrada - GEM A, Niterói, Rio de Janeiro;

- Comissões de prevenção de maus-tratos à criança e ao adolescente no PSF e Rezas, raízes e soro, M aranguape, Ceará.

As categorias analíticas utilizadas para a seleção dos casos e para a elaboração inicial do pro- tocolo de pesquisa foram previamente discutidas em duas oficinas com membros da OPS e do M inistério da Saúde e testadas em piloto realizado em três projetos desenvolvidos por equipes dePSF em Belo H orizonte, M G, selecionadosem mostra local de saúde da família.

A pesquisa foi feita através de análise temática de conteúdo documental, de observação participante e, principalmente, com base em entrevistas realizadas com membros das equipes, população adscrita e outros atores importantes em cada experiência.

A real ização da pesquisa de campo se deu em doismomentos articulados econsecutivos. 0 primeiro momento objetivou a crítica dos conceitos e instrumentos utilizados, com a incorporação do conhecimento gerado pel o contato dos pesquisadores com as práticas pesquisadas, resultando na revisão conceitual e do protocolo de pesquisa adotado no segundo momento, quando se ampliou e aprofundou a pesquisa dos casos.

0 artigo expõe a revisão teórica e a resultante estrutura conceitual desenvolvida na pesquisa. N ão é cabível realizar aqui, portanto, qualquer detaIhamento das experiências pesquisadas nem do protocolo adotado; os interessados podem obter estas informações no relatório final da pesquisa ${ }^{1}$.

Reiteramos, no entanto, que a estrutura conceitual adotada na pesquisa foi primeiramente delineada na revisão bibliográfica e nas oficinas iniciais, constituindo-seno critério de seleção dos casos estudados eorientou a real ização da pesquisa decampo, esendo, por fim, submetida a sucessivas revisões no sentido de absorver o conhecimento gerado no curso da pesquisa de campo.

\section{Definindo promoção da saúde}

Em uma aproximação imediata do termo, podese entender por promoção da saúde toda atuação que tem como objetivo o incremento ou a melhoria da saúde das pessoas.

Não é, no entanto, esse o conceito que se vê apresentar nas práticas que se postulam e são reconhecidas como de promoção da saúde. Nas últimas décadas, a promoção da saúde tem se constituído num campo institucional próprio que reivindica para si um conjunto mais ou menos específico de ações. Essas ações buscariam, efetivamente, o incremento da saúde de um universo humano determinado. É o que se lê, por exemplo, nos dois relatórios do Ministério da Saúde canadense que se inserem na série de documentos genericamente referidos na historio- 
grafia da promoção da saúde, os famosos relatórios Lalonde e Epp 5 . 0 objetivo posto por Lalonde era de "ampliar a liberdade de doença e incapacidade, assim como promover um estado debem-estar suficientepara realizar atividade física, mental esocial". Epp, doze anos depois, postula que "nossa responsabilidadeúltima é garantir que a saúde dos canadenses seja protegida e ampliada". Mas, as ações de promoção da saúde teriam características distintas de outras com 0 mesmo objetivo.

Para tentar delinear essas características, vamos fazer um rastreamento sintético do desenvolvimento do campo teórico e prático de promoção da saúde. Partimos da afirmação de um sentido positivo para a saúde, como algo além da ausência de doenças que, como todos sabem, consta da Constituiçã̃o da Organização Mundial de Saúde, de $1948^{6}$. Em consonância com essa definição, é possível se pensar em um campo de atuação que se volta para a saúde em seu sentido positivo egeral, enão focaliza a recuperação, tratamento ou prevenção de qualquer doença ou agravo específico. Segundo Czeresnia ${ }^{7}$, éo que se lê em "The concept of health and the difference between prevention and promotion" de 1976, quando Leavell \& Clarck escreveram que as medidas de promoção da saúde "não são dirigidas para uma dada doença ou desordem, mas servem para aumentar a saúde geral e o bem-estar".

Nas décadas seguintes, se testemunhou grande desenvolvimento na tecnologia e especialização médica, mas também se percebeu uma dissociação potencialmente explosiva entre os custos aí envolvidos e os resultados em termos de saúde pública. 0 Relatório Lalonde, por exemplo, identifica que a grande maioria dos gastos em saúde estão focalizados na organização da assistência à saúde, 0 denominado setor ou sistema de saúde, enquanto as principais causas de doenças estariam en raizadas nos outros el ementos que o então ministro da saúde canadense incluiu no conceito de campo de saúde, especificamente: biologia humana, ambienteeestilo de vida. O relatório conclui, portanto, que "é aparente que vastas somas estão sendo gastas tratando doenças que poderiam ter sido prevenidas". Alternativas sistêmicas passaram a ser buscadas, como a promoção da saúde e, principalmente, a perspectiva da atenção primária ou básica em saúde, cristalizada na Conferência Internacional sobre Cuidados Primários em Saúde, realizada em Alma-Ata, 1978.

Paralelamente, amplia-se o reconhecimento deque, ao regredirmos nas séries causais de qual- quer patologia ou grupo patológico, vamos encontrar determinações cada vez mais genéricas, incidentes também sobre outras séries causais determinantes de outras patologias. À medida quedistanciamo-nos das causas diretas, proximais da doença pode haver um decréscimo na certeza causal econsistência, com freqüência acompanhada de complexidade crescente. Por outro lado, causas distais provavelmente têm efeitos amplificados elas podem afetar muitos conjuntos diferentes de causas proximais e portanto podem fazer grande diferençå.

Essa compreen são certamenteembasa a concepção de que a atuação sobre esses determinantes mais gerais é potencialmente mais eficaz para o incremento da saúdepopulacional do queações direcionadas para a preven ção de riscos ou o tratamento e a recuperação de agravos específicos, exatamente porque seu impacto se manifestaria como ampliação da saúde, consistindo em proteção contra todo um largo espectro de possíveis patologias e agravos. Essa concepção se complementa com aquelaqueidentifica maior potencial para efetividade em ações direcionadas para riscos atinentes a toda a população ou a vastos grupos populacionais, em vez daquelas direcionadas para riscos detectados em grupos mais restritos. Tal convicção se fortaleceu sobremaneira com o trabalho seminal de Geoffrey Rose ${ }^{9}$, em que se demonstra como fatores de risco comuns a toda uma população podem ter mais efeito sobre a carga de doença nessa mesma população em relação a grupos populacionais específicos submetidos a níveis de risco mais elevados.

Por fim, também se reconhece que as condições sociais são a base para o padrão sanitário de um povo, assim como a posição de cada indivíduo na sociedade é um determinante fundamental da própria saúde. Ao retrocedermos nas séries causais dos princi pais grupos patológicos, daqueles que têm maior magnitude e transcendência nas diversas sociedades, encontramos, entre os determinantes finais, com grande peso, as condições sociais de vida. A qualidade, o padrão das relações sociais resulta, portanto, em elemento com ampla e inquestionável determinação sobre a saúde. 0 reconhecimento dessa evidência respalda o postulado correspondente de alta eficácia potencial da intervenção nas relações sociais para o incremento da saúde.

0 parágrafo anterior contém uma afirmação da determinação social dos indivíduos, inclusive em sua saúde. Façamos aqui uma brevíssima revisão histórica dessa vertente analítica. A apreensão ontológica, ou antropológica, dos in- 
divíduos humanos na interação social, vale dizer, dequeosindivíduos se constituem na redesocial ao mesmo tempo em que são constituintes da redesocial em que vivem, éal go que se consolida no pensamento social, com diversos matizes e inflexões, desde o início do século XIX, com a obra de $H$ egel. A percepção de que as condições sociais têm papel determinante nas condições de saúde das populações afirma-se insistentemente, pelo menos desde a revolução industrial, manifestando-se em um sem-número de artigos e livros em todo o século XX. A partir dos anos oitenta, uma corrente epidemiológica tem evidenciado essa determinação e contribuído para 0 esclarecimento deseus mecanismos psicofisiológicos (referimo-nos aqui à epidemiologia dos determinantes sociais da saúde, quetem a figura de $M$ ichael $M$ armot como a maior referência e os estudos W hitehall como o principal esteio). A principal contribuição dessa epidemiologia tem sido trazer evidência para presença do el o psíquico nas cadeias de determinação social da saúde, demonstrando também sua crescente importância na contemporaneidade. Traduzimos isto nos seguintes termos: o modo de ordenamento social e as posições recíprocas dos indivíduos na hierarquia social determinam largamente o modo como as pessoas se interacionam na sociedade, 0 que, por sua vez, determina as suas condições psíquicas, e isto constitui um elo básico nas cadeias causais de todos os principais agravos e patologias da contemporaneidade.

Chegados ao fim desse brevíssimo percurso sobre as bases teóricas e perspectivas práticas da promoção da saúde, lembramos que ele não pretende, obviamente, ser exaustivo como relato histórico, mas sim significativo como reconstituição conceitual.

\section{Autonomia como categoria norteadora da promoção da saúde}

O conjunto conceitual acima descrito sintetizase coerentemente em uma proposição prática: a ampliação do controle ou domínio dos indivíduos e comunidades sobre os determinantes de sua saúde. I dentificamos este norte para as ações de promoção da saúdeexplícita ou implicitamente desdeo documento conclusivo da Primeira Conferência Internacional de Promoção de Saúde organizada pela OM S em 1986, a Carta de Ottawa ${ }^{10}$ (genericamente reconhecida como marco fundante da promoção da saúde como campo institucional de prática e reflexão sobre a saúde pública): Promoção de saúdeéo processo de capacitação das pessoas para aumentar seu controle sobre emelhorar a sua saúde. Para atingir um estado de completo bem estar físico, mental e social, um indivíduo ou grupo deve ser capaz de identificar e realizar aspirações, satisfazer necessidades etransformar ou lidar com os ambientes. Saúde é, portanto, vista como um recurso para a vida cotidiana, não o objetivo da vida. Trata-se de um conceito positivo enfatizando recursos sociais e pessoais, assim como capacidades físicas. Portanto, promoção de saúde não é apenas responsabilidade de um setor evai além dos estilos devida saudáveis para o bem-estar.

Traduzindo essa proposta, afirmamos que promoção da saúde é a atuação para a ampliação do controle ou domínio dos indivíduos e comunidades sobre os determinantes de sua saúde. Identificamos aí 0 eixo das ações promotoras de saúde, o que nos permite localizar a autonomia como categoria norteadora da atuação em promoção da saúde. Podemos dizer, portanto, que a promoção da saúde busca ampliação da autonomia de indivíduos e comunidades; esseé, a nosso ver, o cerne da proposição de empowerment individual e coletivo.

Mas, o que queremos dizer com ampliação da autonomia?

O conceito de autonomia possui, todos sabem, longa tradição. Não se pretende, de modo algum, dar conta aqui de todas as suas implicações sociais, éticas e políticas, mas também não podemos nos furtar ao debate conceitual dos postulados aqui desenvolvidos.

A idéia de autonomia (auto= próprio, nomos= norma, regra, lei) conduz o pensamento imediatamente à idéia de liberdade e de capacidade de exercício ativo de si, da livre decisão dos indivíduos sobre suas próprias ações e às possibilidades e capacidades para construírem sua trajetória na vida. Não é difícil localizarmos aqui traços do conceito clássico defelicidadena tradição filosófica grega, que identifica a perspectiva da eudaimonia (felicidade, boa fortuna ou equilíbrio, excelência (eu) de sua potência (daimon) com a capacidade do indivíduo para decidir sobre suas próprias ações. Esses são, de fato, valores genericamente propostos nas grandes tradições morais da antiguidade e adensados no estoicismo e epicurismo. Sintetizam-se, enfim, na prescrição: "sê senhor de si".

Essa prescrição parece ter uma forte correspondência com necessidades humanas fundamentais, necessidades de livre expressão de seu ser, ou de manifestação ativa de si, como Marx 
dizia (essa perspectiva atravessa a obra marxiana; para os termos acima utilizados, ver $M$ arx e Engel $\left.s^{11}\right)$. N ecessidade fundamental para os indivíduos humanos que é condição para sua saúde, para sua vitalidade.

Pode-se dizer que essa prescrição é tornada princípio ideológico fundamental euniversalizada na modernidade capitalista, assumindo, nas teorias correspondentes, a forma de condição natural do homem: o princípio de independência original, natural, detodos os indivíduos. Essa concepção antropológica expressa a aparente liberdade individual conquistada com a extensão das relações mercantis a todas as esferas da sociedade, o que é uma característica definidora da forma social capitalista. 0 mercado parece ser a afirmação viva da liberdade individual. Complementarmente, tanto a prática quanto a reflexão políticas modernas encaminharam-se para a afirmação da democracia como o poder coletivo fundado na autonomia individual. Por fim, nos marcos da ordem democrática, o conceito de tal poder deve incorporar o direito à diversidade, impondo-se os limites das liberdades civis, a tolerância eo respeito à livreexpressão de idéias eà palavra públicae, atémesmo, àliberdadedecomportamento de indivíduos e grupos, nos limites do dano a terceiros.

A autonomia, assim considerada, pareceidentificar-se com as relações sociais e todo 0 orde namento social estabelecido sobrea livrecompetição entreindivíduos naturalmenteindependentes. As suas virtudes e os seus limites são aqueles que todos conhecemos.

Talvez por isso, alguns lêem a promoção da saúde como uma corrente derivada do chamado neoliberalismo. Deve-se reconhecer que reforça essa visão a existência de agendas de estímulo à mudança de estilo de vida modeladas sob a pretensão da livre escolha de indivíduos isolados.

No entanto, entende-se como certo quea proposta da promoção da saúde não se esgota em limites liberais. Retornando à Carta de Ottawa, aí lemos que o ativismo em promoção da saúde dirige-se:

. à elaboração de uma política pública sã;

- à criação de ambientes saudáveis: geração de condições detrabalho e vida agradáveis, seguras e estimulantes;

. ao reforço da ação comunitária: participação efetiva e concreta da comunidade na fixação de prioridades, na tomada de decisões e na el aboração eimplantação de estratégias de planificação para alcançar um melhor nível de saúde. A força motriz deste processo provém do poder real das comunidades, da posse edo controleque tenham sobre seus próprios esforços e destinos;

. ao desenvolvimento de aptidões pessoais: favorecer o desenvolvimento pessoal esocial proporcionando informações, educação sanitária e aperfeiçoando as aptidões pessoais indispensáveis para a vida.

. à reorientação dos serviços de saúde, de tal forma que transcenda a mera responsabilidade de proporcionar serviços clínicos e médicos. Esses serviços devem tomar uma nova orientação que seja sensível e respeite as necessidades culturais dos indivíduos.

Essa agenda de promoção da saúde surge em conjunção histórica com outras agen das no contexto das Nações Unidas e com a implantação do SUS no Brasil. Mais do que uma superposição histórica, identificamos aí algumas conjunções ideológicas. Não édifícil perceber correspondências nos objetivos e meios propostos pelas agendas internacionais de desenvolvimento humano, atenção básica em saúde, promoção da saúde e desenvolvimento sustentável, dentre outras, das diversas agências da ONU ${ }^{12}$. Essa identidade é vista também com princípios fundamentais do PSF e do SUS, no Brasil ${ }^{13}$.

São tópicos dessa "agenda comum":

. compromisso com o bem-estar, a qualidade de vida, da população presentee das gerações futuras;

. eqüidade como meta e como meio para a qualificação da vida;

- ampliação das capacidades e possibilidades de escolha individuais e coletivas;

. inclusão social de "minorias";

- afirmação da diversidade e do multiculturalismo;

. atuação comunitária edesenvolvimento deinstrumentos de participação popular deliberativa.

Ainda que se questione o desdobramento da agenda internacional de promoção da saúde desde Ottawa, parece claro que ela não pode ser reduzida à doutrina liberal nem ao discurso neoliberal. Parece mesmo haver grande convergência nesse campo ideológico para o reconhecimento de que a alteridadeéconstitutiva do ser edo realizar-se de cada indivíduo humano. É, portanto, à autonomia construída e realizada na teia de interação e atividade social que se refere aí.

Note-se que, sob esse prisma, reconhecemos a ampliação das possibilidades e capacidades de escolha como promoção da saúde e como desenvolvimento humano.

Em síntese, o que estamos dizendo é que durante a vida nos desenvolvemos - recebemos, re- 
produzimos, desenvolvemos e criamos meios de realização - enos realizamos, de uma maneira ou de outra. 0 indivíduo, de acordo com sua posição no ordenamento social, tem acesso aos meios produzidos e disponibilizados pela humanidade, realizando suas potências naturais em conformidade com o meio social em que vive, e realizando também aquelas potências deorigem propriamentesocial, reproduzidas edesenvolvidas no seu meio social específico. A realização desi dos indivíduos constitui, por sua vez, os momentos ativos do desenvolvimento humano genérico. Assim, ao efetivar as suas potências, o indivíduo éum momento do desenvolvimento da humanidade, o qual não existesem essa realização desi dos indivíduos. $\mathrm{Na}$ efetivação e desenvolvimento de si, os indivíduos realizam e desenvolvem grupos humanos e, de um modo ou de outro, a própria humanidade. Então, para os próprios indivíduos, que são concentrações vivas e pontos de interação da rede social, o desenvol vimento da sociedade resulta em novas condiç̧̃̃es de ser, novas condições de sua existência, inclusivepsicofisiológica.

Pode-seconcluir, portanto, quea saúdeécondição parcial para a realização desi dosindivíduos e meio para o desenvolvimento humano, ao mesmo tempo que este é condição para a saúde. Essa real idade podeser expressa na forma deduas funções de interdeterminação positiva, com 0 pólo predominante posto na ampliação das capacidades humanas e das possibilidades de escoIha dos indivíduos.

Essa percepção tem longa história dentro da análise social eeconômica, sendo marcantea contribuição de Gunnar M yrdal14, em seus estudos sobre o desenvolvimento de regiões ou extratos sociais empobrecidos.

Não é mesmo de se estranhar, pois, que uma forte identidade de propósitos entre a promoção da saúde e o desenvolvimento humano, como institucionalizados no âmbito da O rganização das Nações Unidas, se manifeste desde seus princípios. De acordo com o grande fomentador do PNUD, M ahbub UI Haq ${ }^{15}$,"o princípio básico do desenvolvimento é ampliar as escolhas dos indivíduos". Essa é, como vimos, uma das possíveis traduções do conceito de promoção da saúde expresso desde Ottawa. $\mathrm{N}$ as palavras de Czeresnia ${ }^{16}$, promoção da saúde é o "fortalecimento da capacidade individual e col etiva para lidar com a multiplicidade dos condicionantes da saúde", razão pela qual propõe o "fortalecimento da saúde por meio da construção da capacidade de escolha".

Cumpre advertir, por fim, que a ampliação da compreensão dos determinantes da saúde, em especial a crítica à concepção medicalizada, ou essencialmente biomédica dessa determinação, em favor da percepção do grande peso dos determinantes sociais, não deve conduzir a uma expansão indevida do objetivo das intervenções para a saúde, que turva em vez de ampliar o objeto.

Entendemos que, dentro do amplo escopo do desenvolvimento humano, o campo institucional da saúde, a promoção da saúde inclusive, tem como objeto específico o incremento da capacidade orgânico-psíquica das pessoas. Essa éa sua responsabilidade institucional.

E é com este foco que identificamos a autonomia como a categoria norteadora da promoção de saúde, porque vemos que ela é um determinante positivo fundamental para a saúde, sua ampliação é protetora à saúde assim como, inversamente, a restrição de autonomia é fator de risco elementar na cadeia causal dos principais grupos de agravos e patologias (o que tem sido evidenciado pela epidemiologia dos determinantes sociais).

Para concluir, consideraremos sinteticamente como os conceitos acima desenvolvidos seaplicam à prática de promoção da saúde na atenção básica, que foi o objeto de nossa pesquisa.

Assumimos a autonomia como categoria norteadora e conteúdo central da promoção da saúde que perpassa todos os processos, todas as categorias práticas pelas quais ela se realiza. Por outro lado, dentro do escopo de atuação do setor saúde, entendemos como autonomizadora toda intervenção que amplie a capacidade das pessoas de agirem sobre os determinantes de sua saúde.

Como exposto, a existência dos indivíduos apenas ocorre e pode ocorrer em sua interação, isto é, através de grupos, compondo coletivos humanose, por fim, dentro do coletivo geral que constitui a sociedade em que vivem. Portanto, 0 desenvolvimento da autonomia e os mecanismos para tal apenas podem ocorrer através da comunidade. Parece claro, portanto, queaqueles mecanismos e instrumentos que ampliem capacidades deintervenção coletiva terão mais sucesso na ampliação da autonomia do que aqueles que apenas sejam focalizados no indivíduo.

A atuação em atenção básica em saúde, assim como toda atuação local, tem claros limites no que toca a esta ampliação da autonomia no coletivo, porqueas condições locais são articuladas num sistema social e, no limite, a ele submetidas. Cultura, formas de trabalho e distribuição social de recursos, por exemplo, não são predominantementeescolhidos em cada coletivo local. 
Ainda assim, as atuações no nível local podem ampliar as possibilidades de escolha e deliberação dos indivíduos sobre os diversos determinantes desua saúde. M ecanismos de deliberação participativa e formas de atuação social, re des de proteção e cuidado recíproco, respeito à diversidade de comportamentos, etc. são exemplos de processos autonomizantes que podem ser desencadeados no âmbito local. Esse foi 0 grande aprendizado que extraímos das experiências pesquisadas.

A prática da atenção básica em saúde pode, portanto, ser promotora de saúde de vários modos e por vários mecanismos, entre eles:

- ampliação do acesso popular ao conhecimento sobre os determinantes da saúde e sobre os mecanismos e processos para intervenção sobre os determinantes da saúde;

. implemento do autocuidado e doscuidados com dependentes: capacitação e desenvolvimento, por parte dos indivíduos da comunidade local, de práticas reconhecidas como de cuidado com a saúde, própria e de dependentes, como crianças e idosos, e de comportamentos reconhecidos como saudáveis;

- participação popular deliberativa: implantação edesenvolvimento de mecanismos efetivos de deliberação popular;

. inclusão social:

- atuação coletiva para ampliar o acesso a bens e serviços que permitam a intervenção dos indi víduos da comunidade sobre os determinantes de sua saúde e o desenvolvimento de comportamentos reconhecidos como saudáveis. Acesso à educação e bens culturais, renda, melhores condições de trabalho, áreas de lazer e preservação ambiental, atividades lúdicas e de convivência, meios de transporte, etc.;

- absorção social de grupos excluídos;

- redes sociais de proteção e cuidado recíproco: implantação e desenvolvimento deiniciativas deproteção ecuidado recíproco na comunidade. Implantação e desenvolvimento de mecanismos deinteração entre as diversas iniciativas neste sentido no território em questão.

Para finalizar, podemos afirmar, em coerência com todo o raciocínio acima exposto, que as práticas e os processos que propiciem a participação ativa edeli berativa dos sujeitos envolvidos são essenciais em todos os mecanismos de promoção da saúde, tanto na atuação junto aos diversos níveis de coletividade, quanto na clínica individual.

Lembramos que o referencial teórico aqui exposto foi fruto de revisões bibliográficas sucessi- vas e de oficinas de trabalho realizadas durante a pesquisa e também do confronto dos conceitos oriundos deste processo com as práticas pesquisadas. Pudemos verificar que a perspectiva de ampliação da autonomia individual e col etiva dos sujeitos envolvidos estava fortementepresentenas experiências analisadas, mesmo que esse referencial teórico não se apresentasse conscientemente para os principais protagonistas. De fato parte relevante das práticas pesquisadas não se considerava nem se autodenominava de "promoção da saúde", apenas procurava enfrentar os reais determinantes da saúde da população adscrita.

Identificamos queo impulso ao enfrentamento dos determinantes da saúde, das causas das causas, encontra-se latente no modelo de atenção primária à saúde do PSF; pode-se esperar, portanto, que a difusão do referencial teórico aqui exposto junto à comunidade do PSF no país possa contribuir para a ativação de práticas efetivas de promoção de saúde neste universo.

\section{Colaboradores}

P Fleury-Teixeira coordenou a pesquisa eas revisões bibliográficas, participou da orientação, redação e revisão do relatório final da pesquisa. FAC Vaz participou das discussões conceituais, metodológicas e colaborou na fase deanálise dos dados e na construção do relatório final. FCC Campos participou da coordenação do projeto, da elaboração do projeto de pesquisa e da orientação e redação da análise e relatório final. J Alvares participou das discussões sobre marco teórico, metodologia, construção das categorias de análise, redação e revisão do relatório final. RAT Aguiar e VA Oliveira participaram das discussões sobre marco teórico, metodologia, construção das categorias de análise, redação e revisão do relatório final. 


\section{Referências}

1. Nescon. Faculdade de M edicina. UFM G. Disponível em: http://dtr2004.saude.gov.br/dab/caadab/ documentos/promocao_saude_ab.pdf.

2. Associação Brasileira de Pós-Ğraduações em Saúde Coletiva. Livro de Resumos. Cien Saude Colet 2003; 8(Supl 2).

3. Brasil. M inistério da Saúde. Secretaria de Atenção à Saúde. Departamento de Atenção Básica. II M ostra Nacional de Produtos de Saúde da Família: trabaIhos premiados. Brasília: M inistério da Saúde; 2006

4. Lalonde M. A N ew Perspective on the Health of $\mathrm{Ca}$ nadians: a Working Document. Ministry of $\mathrm{Heal}$ th and Welfare, Ottawa, Canada, 1974. [acessado 2005 Fev 14]. Disponível em: http://www.hcsc.gc.ca/hcs-sss/com/lalonde/index_e.html

5. Epp J. Achieving Health For All: A Framework For $\mathrm{H}$ ealth Promotion. Ottawa: M inistry of $\mathrm{H}$ ealth and Welfare; 1986.

6. Organização Mundial de Saúde. Preamble to the Constitution of the World $\mathrm{H}$ ealth Organization as adopted by the International $\mathrm{H}$ ealth Conference, $\mathrm{New}$ York, 19-22 June, 1946; signed on 22 July 1946 by the representatives of 61 States. Official Records of the World Health Organization, no. 2, p. 100 and entered into force on 7 April 1948. [acessado 2005 Ago 08]. Disponível em: http://www.who.int/about/ definition/en/

7. Czeresnia D, Freitas CM, organizadores. Promoção da Saúde: reflexões, conceitos, tendências. Rio de Janeiro: Fiocruz; 2003.

8. Organização Mundial de Saúde. World Health Report 2002: reducing risks, promoting healthy life. Genebra, 2002. [acessado 2005 Mar 16]. Disponível em: http:/ /www.who.int/whr/previous/en/index.html

9. Rose G. Sick Individuals, Sick Populations. Int J Epid. 1985; 14(1):32-38.
10. Organização Mundial de Saúde. Ottawa Charter for $\mathrm{H}$ ealth Promotion. First International Conference on Health Promotion. Ottawa, 21 N ovember 1986 WHO/HPR/HEP/95.1. [acessado 2005 Ago 07]. Disponível em: http://www.who.int/hpr/NPH/docs/ ottawa charter hp.pdf

11. Marx K , Engels $\mathrm{F}$. A ideologia alemã (Feuerbach). São Paulo: Hucitec; 1996.

12. Aguiar RAT. A construção internacional do conceito de atenção primária à saúde (APS) e sua influência na emergência e consolidação do Sistema Ú nico de Saúde no Brasil. [dissertação]. Belo Horizonte (M G): Universidade Federal de M inas Gerais; 2003.

13. Ferreira JR, Buss PM. Atenção Primária e Promoção da Saúde. In: Brasil. Ministério da Saúde. Projeto Promoção da Saúde. Promoção da Saúde. Brasília: Ministério da Saúde; 2001. p. 7-14.

14. Myrdal G. Teoria econômica e regiões subdesenvolvidas. Belo Horizonte: Editora da UFM G; 1960.

15. Organização das Nações Unidas. Programa de Desenvolvimento Humano (PNUD). What is Human Development. Versão eletrônica. [acessado 2005 Abr 14]. Disponível em: http://hdr.undp.org/hd/

16. Castiel LD. Dédalo e os dédalos: identidade cultural, subjetividade e os riscos à saúde. In: Czeresnia $D$, Freitas CM , organizadores. Promoção da saúde. Conceitos, reflexões, tendências.1aㅡ ed. Rio de Janeiro: Fiocruz; 2003. p. 79-95.

Artigo apresentado em 24/10/2006

Aprovado em 05/07/2007

Versão final apresentada em 18/09/2007 